\title{
Predator-Mediated Plasticity in Morphology, Life History, and Behavior of Daphnia: The Uncoupling of Responses
}

\author{
Maarten Boersma, ${ }^{1, \star}$ Piet Spaak, ${ }^{1, \dagger}$ and Luc De Meester ${ }^{1,2,}$
}

1. Max-Planck-Institut für Limnologie, Postfach 165, D-24302 Plön, Germany;

2. Laboratory of Ecology and Aquaculture, Katholieke Universiteit Leuven, Naamsestraat 59, B-3000 Leuven, Belgium

Submitted October 9, 1997; Accepted January 21, 1998

AвSTRAст: We studied the way 12 traits responded to fish kairo-
mones in a set of 16 Daphnia magna clones derived from four dif-
ferent habitats-two where daphnids co-occur with fish and two
without fish. These clones differed widely in their response to
predator kairomones, with none of the clones showing a signifi-
cant response in all traits and all clones showing a response for at
least one trait. Most of the clones showed a significant response in
one to four traits, with no evidence for an association between dif-
ferent traits. Clones from fish habitats were slightly more respon-
sive to the presence of fish kairomones than clones from fishless
locations. We conclude that most clones show an induced re-
sponse to the presence of their predators (fish) but that there is a
large genetic variability with respect to the traits for which clones
show a response. Our results indicate that the major distinction is
not between inducible and noninducible genotypes but rather that
the genotypes differ in the combination of traits for which they
show inducible responses.

Keywords: Daphnia, fish predation, inducible defense, kairomone.

Phenotypic plasticity can be defined as environmentally induced variation, leading to the expression of different phenotypes by a given genotype. Phenotypic plasticity may be the result of developmental noise or may involve systematic and repeatable responses to environmental cues. A wide variety of organisms have been shown to respond to environmental changes and to produce adaptive phenotypes (e.g., Stearns 1989). Many of these changes in

*E-mail: boersma@mpil-ploen.mpg.de.

$\dagger$ Present address: Department of Limnology, EAWAG/ETH, Überlandstrasse 133, CH-8600 Dübendorf, Switzerland; E-mail: spaak@eawag.ch.

‡E-mail: luc.demeester@bio.kuleuven.ac.be.

Am. Nat. 1998. Vol. 152, pp. 237-248. (C) 1998 by The University of Chicago. 0003-0147/98/5202-0006\$03.00. All rights reserved. phenotype are a direct response to the presence of predators, and the role of this chemical communication between predators and their prey has been the subject of a large number of studies (reviewed by Tollrian and Harvell 1998). Especially in the last decade, it has become increasingly clear that chemicals excreted by predators (kairomones) influence a wide range of characteristics of their prey species. In aquatic environments, predator kairomones have been shown to affect morphology, lifehistory traits, and behavior of prey species (reviewed by Harvell 1990; Larsson and Dodson 1993). These predator-prey communication links have been found throughout aquatic food webs, including the relationship between piscivorous fish and planktivorous fish (e.g., Gliwicz and Jachner 1993; Nilsson et al. 1995), between planktivorous fish and zooplankton (e.g., Stibor 1992; Reede 1995), between invertebrate predators and zooplankton (e.g., Stemberger and Gilbert 1987; Kusch 1993b; Lüning 1995; Tollrian 1995), and between zooplankton and algae (Hessen and van Donk 1993; Lampert et al. 1994).

Species of the genus Daphnia, one of the best-studied genera in freshwater environments, are very plastic in their reaction to chemicals excreted by their predators. Members of this genus have been shown to exhibit predator-induced changes in life-history traits (size and age at maturity, size and number of eggs produced, production of sexual eggs: Spitze 1992; Weider and Pijanowska 1993; Macháček 1995; Ślusarczyk 1995), changes in morphology (helmet development, neck spines: Tollrian 1990; Repka et al. 1995), and changes in behavior (diel vertical migration, phototaxis, swarming and escape reactions: Ringelberg 1991; De Meester 1993; Pijanowska 1994; Kvam and Kleiven 1995). Each of these responses has been separately interpreted as an adaptive mechanism to avoid or to counterbalance predation, and it has been assumed implicitly that daphnids should react with as many different traits as possible. Recently, however, it has been argued (De Meester and Pijanowska 1996) that it is not obvious that it would be adaptive to exhibit all responses at the same time. It has, for instance, been ob- 


\section{The American Naturalist}

served that there is an association between diel vertical migration (phototactic behavior) and life-history traits, with larger bodied genotypes remaining at greater depth during the day than smaller ones (De Meester 1994). Although both diel vertical migration and a reduced body size lower the probability of being detected by visually hunting predators, it may often not be adaptive to decrease size at maturity while engaging in a diel vertical migration because food levels in the hypolimnion are typically low and starvation resistance is known to be higher for larger individuals (Threlkeld 1976). A selection experiment (De Meester et al. 1995) with different Daphnia hyalina $\times$ galeata clones isolated from the same lake has shown that the association between life-history and vertical migration traits may result in similar relative fitness of clones in the presence of predators.

Most authors studying the reaction of multiple traits to chemicals exuded by predators have assumed that morphological changes, such as the induction of helmets, neck teeth, and spines, should have costs (Jacobs 1967; Parejko and Dodson 1991) and that induced animals should show changes in life-history traits as a result of these costs. However, several independent studies have shown that, in general, no strong association can be observed between the degree of neck teeth induction and life-history characteristics (Spitze 1992; Black 1993; Lüning 1994; Tollrian 1995). This was interpreted by most of the above-mentioned authors as evidence for the low costs of neck teeth formation. If, however, the responses of morphological and life-history traits to the presence of fish kairomones are independent from each other, the presence or absence of correlated responses would depend on whether a combined response is adaptive or whether it reflects costs of the morphological defense. Results of the few studies that involved two different traits indeed indicate that induced responses in different traits can at least be partly uncoupled (Spitze 1992; Lüning 1994; De Meester and Pijanowska 1996).

Thus far, no studies have been carried out to investigate specifically the degree of coupling of predator-induced responses in different traits. Therefore, we investigated the responses of a number of morphological, behavioral, and life-history traits to the presence of fish kairomones in a set of 16 Daphnia magna Straus clones that were isolated from different locations. Life-history, morphological, and behavioral data were collected to yield a data matrix of 12 traits by 16 clones. Figure 1 shows two of the possible outcomes of this experiment. According to the implicit assumption in many earlier articles on predator-induced responses in which reference is made to inducible and noninducible genotypes, the expectation is that some clones will react to the presence of

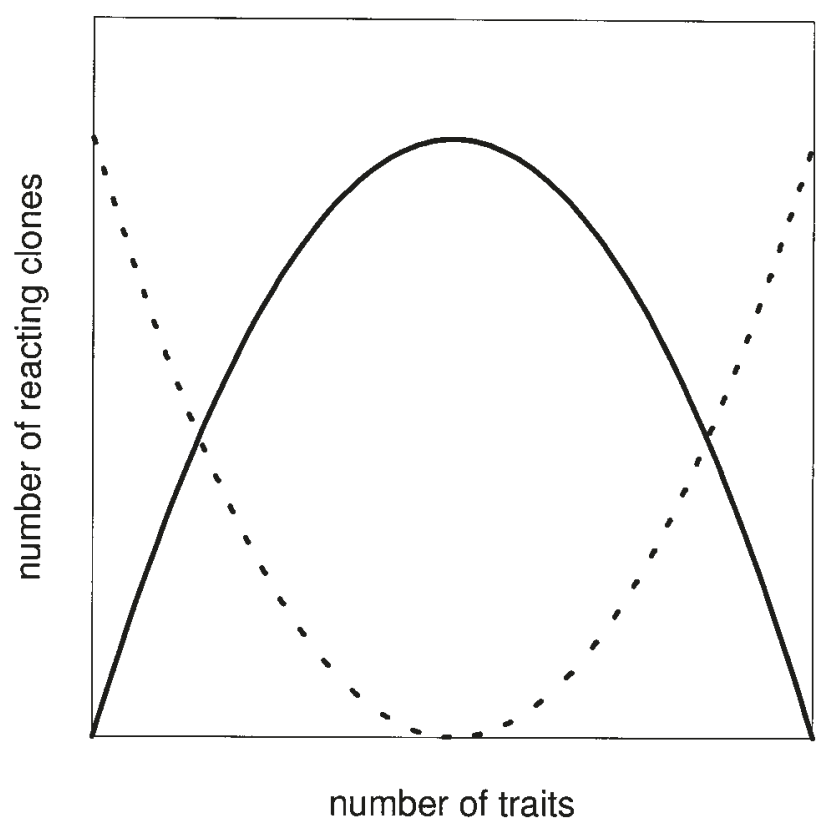

Figure 1: Possible frequency distributions of the number of clones reacting to the presence of fish chemicals in a number of different traits. The solid line indicates the case that all clones react with a subset of the possible traits. The dotted line shows the case where some clones would not react at all (or with a very low number of traits) and others with a very high number of traits (inducible and noninducible genotypes).

fish kairomones with all of the traits under consideration, whereas others do not react with any of the measured traits (fig. 1, dotted line). Alternatively, most of the clones might react with a subset of the traits measured, leading to a curve similar to the solid line of figure 1, with the frequency of clones reacting with all traits or showing no response at all being relatively low. Two factors could influence the skewness of the frequency distribution. If the cumulative cost of exhibiting several induced responses at the same time becomes too high, this will lead to a skewed distribution to the right. The same skewness will be found if selection pressures on the inducibility of other traits decline once an adaptation in one trait has evolved.

Similar patterns should be visible in computed values of genetic correlations between traits. If it is the case that there are inducible and noninducible genotypes, then we would expect not only a high degree of genetic correlation between these traits but, even more important, high genetic correlations between the change in the values of these traits to the presence of predator kairomones as well. Under the hypothesis that the responses of the different traits to the presence of predator kairomones are uncoupled, one expects generally low values for the genetic correlation between the induced changes among 
traits. We further investigated whether suites of traits that tend to react simultaneously to the presence of predator kairomones could be identified. Finally, in our analysis of clones from four different habitats, we expect to find a higher correlation of the responsiveness with respect to a particular trait among clones isolated from the same habitat, as these clones faced more similar selective pressures, than among clones isolated from different habitats.

\section{Material and Methods}

Clonal lineages were established from Daphnia magna populations inhabiting four sites in Germany and Belgium: two lakes with fish present and two ponds that contain no fish. From each population, we worked with four clones. The Belgian clones originated from Lake Blankaart, a shallow hypertrophic lake in which fish, including bream (Abramis brama L.) and roach (Rutilus rutilus L.), are present in relatively high densities, and from Citadelpark, a fishless pond in the city of Ghent. The German clones were collected from the Großer Binnensee, a shallow, slightly brackish, and hypertrophic coastal lake, which contains unknown densities of bream, roach, and perch (Perca fluviatilis L.; Lampert 1991), and from the Rixdorfer Pferdetränke, a small eutrophic fishless pond.

Most Daphnia species are cyclic parthenogens: during the largest part of the growing season, reproduction takes place through ameiotic parthenogenesis, and sexual reproduction occurs relatively seldom. Sexual eggs are resting stages and are protected by a hard case called an ephippium. To ensure that the experimental clones would be genetically distinct, clonal lineages were established from resting eggs, collected from the different habitats. This ensured that the clones taken in this study were a random representation of the gene pool at the start of the growing season. For all but one population, ephippia collected in the habitat were hatched in the laboratory. The clones from Citadelpark hatched in situ. Four clones per location were chosen randomly and cultured in 1-L jars in the laboratory for many generations before the experiments were performed.

Neonates were collected from the stock cultures, and six to 10 individuals were placed into $200-\mathrm{mL}$ jars. These individuals, the grandmothers of the experimental animals, were kept at $20^{\circ} \mathrm{C}$, under a long-day photoperiod (16L:8D) and fed with the green alga Scenedesmus acutus Meyen from chemostat cultures. Algal concentration of the medium was $0.8 \mathrm{mg} \mathrm{C} \mathrm{L} \mathrm{L}^{-1}$, and medium was refreshed daily. Third-brood neonates of these animals were isolated to serve as mothers for the experimental animals and were subjected to the same conditions as their mothers. The neonates from the second brood of these animals were used in the life-table experiment.

The experimental vessels $(200 \mathrm{~mL})$ were stocked with seven neonates per vessel and were subjected to the following treatments in a full factorial design: fish water/ nonfish water, crowded water/noncrowded water, short day/long day. Fish water was obtained from a 40-L aquarium, which contained two fish (Leuciscus idus L.) of approximately $5 \mathrm{~cm}$ in length. Water was collected from this aquarium daily, filtered through a $0.45-\mu \mathrm{m}$ filter, and aerated for $10 \mathrm{~min}$. The aquarium was refilled with unfiltered water from the nearby Lake Schöhsee. The fish were fed daphnids daily, but care was taken that feeding was done in water that was subsequently discharged, such that the fish-conditioned water could not be influenced by Daphnia alarm substances. Crowded water was obtained by filtering water $(0.45-\mu \mathrm{m}$ filter $)$ previously inhabited by a very dense ( $>300$ individuals $\mathrm{L}^{-1}$ ) population of a Daphnia pulex de Geer clone. Daphnia pulex was used for generating crowded water in order to avoid interference from a closer relationship of one of the experimental clones with the animals that conditioned the crowded medium. Moreover, the D. pulex clone used has been shown to affect life histories of D. magna (Hobæk and Larsson 1990). The crowded cultures were resupplied daily with $0.45-\mu \mathrm{m}$ filtered Schöhsee water and fed three to four times daily with $S$. acutus at a concentration higher than $1 \times 10^{6}$ cells $\mathrm{mL}^{-1}$. Fish medium and crowded medium consisted of half conditioned water and half $0.45-\mu \mathrm{m}$ filtered Schöhsee water; the fish medium plus crowded medium consisted of half fish water and half crowded water. All animals were kept at $20^{\circ} \mathrm{C}$

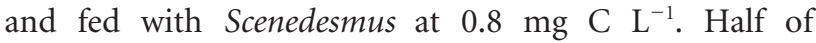
the animals were subjected to a long-day photoperiod (16L:8D), the other half were kept under short-day light conditions (8L:16D). This setup resulted in a total of 2 (day length) $\times 2$ (fish factor) $\times 2$ (crowding factor $) \times 4$ (populations) $\times 4$ (clones) $\times 2$ (replicates $)=256$ experimental vessels. The use of this range of environmental conditions creates a suitable data set for the analysis of responses to fish kairomones as the results obtained from these experiments should have a more general bearing than the mere comparison of animals under conditions with and without fish chemicals.

The following life-history parameters were used in the analysis to contrast traits of animals grown under fish and nonfish conditions: size at maturity, age at maturity, mature growth (size of the fourth adult instar minus size at maturity), number of eggs produced by the first adult instar, number of eggs produced by mature instars $2-4$, size of the newborn produced by the first adult instar, and the percentage of males produced by six adult instars. Three morphological traits, known to affect prey 
vulnerability, were measured. Length of the tail spine at maturity and length of the tail spine of newborns produced by the first adult instar female have been shown to affect handling times by predators (e.g., Swaffar and O'Brien 1996). Eye size affects the visibility of the prey to visual predators (e.g., Zaret and Kerfoot 1975). We measured the size of the eyes of fifth adult instar individuals in animals grown under long-day conditions.

Although several studies have shown that significant phenotypic correlations do exist between some of the morphological and life-history traits studied here (e.g., between size at maturity and size of the neonates; Boersma 1995), the traits listed above were chosen to represent a set of almost independently measurable traits, among which no a priori correlations are expected because no measurements are used in more than one trait. We did not, for example, use the intrinsic rate of population increase, $r$, as a trait because this quantity is computed from the age at maturity and the number of offspring produced. Per clone and per character trait threeway ANOVAs were carried out with fish/nonfish, crowded/noncrowded, and long day/short day as fixed factors, and the significance of the fish factor effect was assessed. For eye diameter, two-way ANOVAs were performed.

Two behavioral traits, phototaxis and escape reaction (alertness), were assessed in separate experiments, contrasting only fish and nonfish conditions. All 16 clones were assessed for their phototactic behavior following earlier research (De Meester 1991). The experimental setup consisted of a glass column, $25 \mathrm{~cm}$ high and $5 \mathrm{~cm}$ internal diameter, filled with dechlorinated tap water. The column was placed in a darkened box and illuminated from above. The vertical distribution of 10 naive test animals was recorded for $10 \mathrm{~min}$, at 1-min intervals. The observations of the second 5 min were averaged in the calculation of the phototactic index. This phototactic index was defined as $(U-L) /(U+M+L)$, in which $U, M$, and $L$ are the number of animals observed in the upper $(12 \mathrm{~cm})$, middle $(10 \mathrm{~cm})$, and lower $(3 \mathrm{~cm})$ compartment, respectively. Three experiments were carried out, each with new animals, yielding three observations per clone and treatment, with the two treatments being the culture of the animals in the absence or presence of fish kairomones (at high food concentration and $20^{\circ} \mathrm{C}$; see De Meester 1991, 1996, for more details). Significance of the phototactic response for each clone was calculated using $t$-tests.

Alertness of the different clones under control conditions and in the presence of fish kairomones was established according to a protocol developed earlier (De Meester and Pijanowska 1996). Populations of 10 adult females per clone were introduced into 1-L beakers. Nine persons, inexperienced in handling Daphnia, were asked to catch the animals with a pipette $(0.4 \mathrm{~cm}$ internal diameter). The time needed to pick up 10 animals individually was recorded to the nearest $0.1 \mathrm{sec}$. All animals used were cultured in 1-L jars and fed a high concentration of S. acutus daily. In order to avoid differences other than the behavioral ones, all animals were cultured in the absence of fish kairomones, and only $12 \mathrm{~h}$ prior to the experiments, half of the animals were transferred to fishconditioned water. Each "predator" sampled each of the 32 populations (16 clones; two treatments) in random order. As predator efficiency was highly variable between persons, we analyzed the data using Wilcoxon paired sample tests, matching the catching efficiency per predator for each clone under control conditions and with fish kairomones present.

All experiments combined resulted in a matrix of 16 clones and 12 character traits per clone (table 1). In our tests, we used a significance level of .05 , and no correction for multiple tests was made, as the often used, but still very conservative, sequential Bonferroni test (Rice 1989 ) would require the smallest $P$ value in this table of 192 tests to be $.05 / 192(=.00026)$ to obtain statistical significance at the .05 level. Given the error and effect variation observed in our experiments, this would mean that we should have taken more than 30 replicates per clone instead of the eight we had now. This would have resulted in a total of around 1,000 experimental vessels, a value clearly outside the range of normal laboratory possibilities. Moreover, we were interested in patterns of reaction and correlated responses, and these patterns will also be visible at lower levels of significance.

Genetic correlations between traits and between the responses of the traits were computed to assess the degree of genetic coupling of the traits. For this, ANOVAs and MANOVAs were carried out per treatment, and the average of the computed genetic correlations was computed over all treatments (Spitze et al. 1991). As the two behavioral traits were not measured in the same animals, the genetic correlations between these traits, and between these traits and the morphological and life-history traits, were computed by random coupling of values for morphology/life history with the behavioral data.

To assess the degree of coupling in the reactions of different traits, we also computed the genetic correlations in the plasticity of the traits. We used the percentage change induced by the presence of fish kairomones as input data for the ANOVAs and MANOVAs (Spitze et al. 1991), through randomly pairing the values obtained from animals cultured in the absence and presence of fish kairomones and otherwise identical conditions. This method, however, most likely involves high variation as a result of the random pairing. Therefore we also performed cluster 
Table 1: Reaction matrix of responses of 16 clones of Daphnia magna to the presence of fish kairomones

\begin{tabular}{|c|c|c|c|c|c|c|c|c|c|c|c|c|c|c|c|c|c|c|}
\hline \multirow[b]{2}{*}{ Trait } & \multirow[b]{2}{*}{ Class } & \multicolumn{4}{|c|}{ Großer Binnensee } & \multicolumn{4}{|c|}{ Lake Blankaart } & \multicolumn{4}{|c|}{ Citadelpark } & \multicolumn{4}{|c|}{ Rixdorfer Pferdetränke } & \multirow[b]{2}{*}{ Significant } \\
\hline & & 13 & $1 B$ & $7 A$ & $7 B$ & 20 & 36 & 46 & 82 & 24 & 29 & 38 & 43 & $2 B$ & 4 & $5 A$ & 7 & \\
\hline Size at maturity & $\mathrm{L}$ & .046 & .030 & $x \times x$ & $x \times x$ & $x \times x \times$ & $x \times x$ & .024 & $x \times x$ & $x \times x \times$ & $x \times x$ & $\times \not x$ & .013 & $x \times x$ & $x \times x$ & $x \times x$ & .047 & 5 \\
\hline Age at maturity & $\mathrm{L}$ & $x \times x$ & $x \times x$ & .002 & $<.001$ & $x \times x$ & $x \times x$ & $x \not x$ & $x \times x$ & $x \times x \times$ & $x \times x$ & $\times x$ & $x \times x$ & $\times \times \times$ & .001 & .022 & $x \times x$ & 4 \\
\hline Mature growth & $\mathrm{L}$ & $x \times x$ & $x \times x$ & $x \not x$ & .017 & $x \times x$ & $x \times x$ & $x \not x$ & $x \times x$ & $\times .009$ & .003 & $\times x$ & $x \times x$ & $x \times x$ & $\times \not x$ & $x \times x$ & $\times \not x$ & 3 \\
\hline Number of eggs at maturity & $\mathrm{L}$ & $x \times x$ & $x \times x$ & $x \times x$ & $x \times x$ & $x \times x \times$ & $x \times \times$ & $x \not x$ & $\times \times \times$ & $x \times x \times$ & $x \times x$ & $\times \not x$ & $x \times \times$ & $x \times x$ & $x \not x$ & $x \times x$ & $\times \not x$ & 0 \\
\hline Size of neonates & $\mathrm{L}$ & .002 & $\times \not x$ & $x \times x$ & .010 & .029 & $x \times x$ & $x \times x$ & $x \times x$ & $x \times x \times$ & $x \times x$ & $\times \not x$ & $x \times x$ & $\times \times \times$ & $x \not x$ & $x \times x$ & $\times \not x$ & 3 \\
\hline Percentage males & $\mathrm{L}$ & .049 & $\times x$ & $x \times x$ & .003 & $x \times x$ & $x \times x$ & $x \not x$ & $x \times x$ & $x \times x \times$ & $x \times x$ & $\times x$ & $x \times x$ & $\times \times \times$ & $x \not x$ & .017 & $\times \not x$ & 3 \\
\hline Spine length adult & M & $x \times x$ & $x \times \times$ & $x \times x$ & .047 & $x \times x$ & $x \times x$ & $x \not x$ & $x \times x$ & $x \times x \times$ & .002 & $\times \not x$ & $x \times x$ & $\times \times \times$ & $x \not x$ & .005 & $\times \not x$ & 3 \\
\hline Spine length neonate & M & $x \times x$ & $x \times x$ & $x \times x$ & $\times \times \times$ & $x \times x \times$ & $x \times x$ & $x \not x$ & $x \times x$ & $x \times x \times$ & $x \times x$ & $\times \not x$ & $x \times \times$ & .008 & $x \times$ & $x \times x$ & $\times \not x$ & 1 \\
\hline Eye diameter & M & $x \times x$ & $x \times x$ & $x \not x$ & .019 & .004 & $x \times x$ & $x \times x$ & $x \times x$ & $x \times x \times$ & $x \times x$ & $x \times x$ & $x \times x$ & $\times \times \times$ & $x \not x$ & $x \times x$ & $x \not x$ & 2 \\
\hline Phototactic behavior & B & .022 & $x \not x$ & $x \times x$ & .046 & .022 & .002 & $<.001$ & $<.001$ & $\times x$ & $x \times x$ & .004 & $x \times x$ & $\times \times \times$ & $x \not x$ & $x \times x$ & .004 & 8 \\
\hline Alertness & B & $x \times x$ & $x \times x$ & $x \times x$ & $\times \times x$ & $x \times x \times$ & $x \times x$ & $\times x$ & $\times \times x$ & $x \times x \times$ & .004 & $\times \not x$ & $x \times x$ & $\times \times \times$ & $x \not x$ & $x \times x$ & $\times x$ & 1 \\
\hline Total significant & & 5 & 1 & 1 & 7 & 4 & 1 & 2 & 2 & 2 & 3 & 1 & 1 & 1 & 1 & 3 & 2 & 37 \\
\hline
\end{tabular}

Note: Ellipses indicate $P$ values $>.05$; for significant $(P<.05)$ changes the exact $P$ values are given. The critical $\alpha$ values for Bonferroni corrections are $0.05 / 12=$ 0.004 , assuming 12 (per clone) nonindependent tests or $0.05 / 192=0.00026$, when correcting for all tests. The traits are grouped into life history (L), morphological $(\mathrm{M})$, and behavioral (B) traits.

analysis on the data (complete linkage, Euclidean distances) and analyzed whether we would find suites of responses - that is, do some clones react with a certain set of traits whereas others react with other ones?

Discriminant analysis was carried out to assess whether there were significant differences in the reactions of clones isolated from different habitats - that is, do clones isolated from locations where fish are present react with a different set of traits to the presence of fish chemicals than clones isolated from fishless lakes? Both the discriminant analysis and the cluster analysis were carried out on the measured differences between the animals under fish and nonfish conditions, and the data were standardized before being used in these analyses.

\section{Results}

All but one of the traits under study showed a significant reaction in at least one of the clones, but none of the traits was significantly affected by the presence of fish factor in all of the clones tested (table 1). All clones reacted with at least one trait, but none reacted with a shift in all of the traits studied. The reaction norms of the 16 clones for the 12 traits are shown in figure 2. Solid lines indicate significant differences between fish and nonfish conditions. Figure 2 shows that, within traits, the direction of most of the significant responses is the same. The maximum number of traits showing a significant reaction to fish chemicals in a given clone was seven out of 12, for one clone collected from the Großer Binnensee (table 1). Phototactic behavior and size at maturity were the most responsive traits. Figure 3 summarizes these results and shows the frequency distribution of the number of significant traits per clone. The comparison of figures 1 and 3 shows that the solid line of figure 1 describes our actual results more accurately than the dotted line, but it also becomes clear that the actual frequency distribution is heavily skewed to the right.

Even most clones isolated from fishless environments (Citadelpark and Rixdorfer Pferdetränke) exhibited a significant reaction to fish kairomones for at least one trait and, thus, can recognize the chemical presence of fish. Although there was a tendency for the clones isolated from fishless environments to be less responsive (averaging 1.75 responsive traits per clone) than clones isolated from habitats with fish (2.88 significant traits per clone), this difference was not significant (Mann-Whitney $U$ test, $U=22 ; P=.27$ ).

The relatively low percentage of significant genetic correlations between different traits (19 from a total of 66, or $29 \%$ ) shows that the link between the different traits was not very strong (table 2 ). This suggests that the traits are largely uncoupled. As a result of the necessity to couple different individuals to be able to compute genetic correlations in the changes in traits, the variation, as computed by the jackknifing technique described by Meyer et al. (1986), was large, and none of the genetic correlations was significantly different from 0 . Moreover, four of the traits showed negative genetic variances, and, hence, it was not possible to compute genetic correlations with these traits. Cluster analysis (fig. 4) revealed three major groups, none of which is exclusively characterized by one type of trait (morphology, life history, behavior), although the two behavioral traits clustered together. The first cluster can be best described as "adult" characteristics, the second one is a combination of 

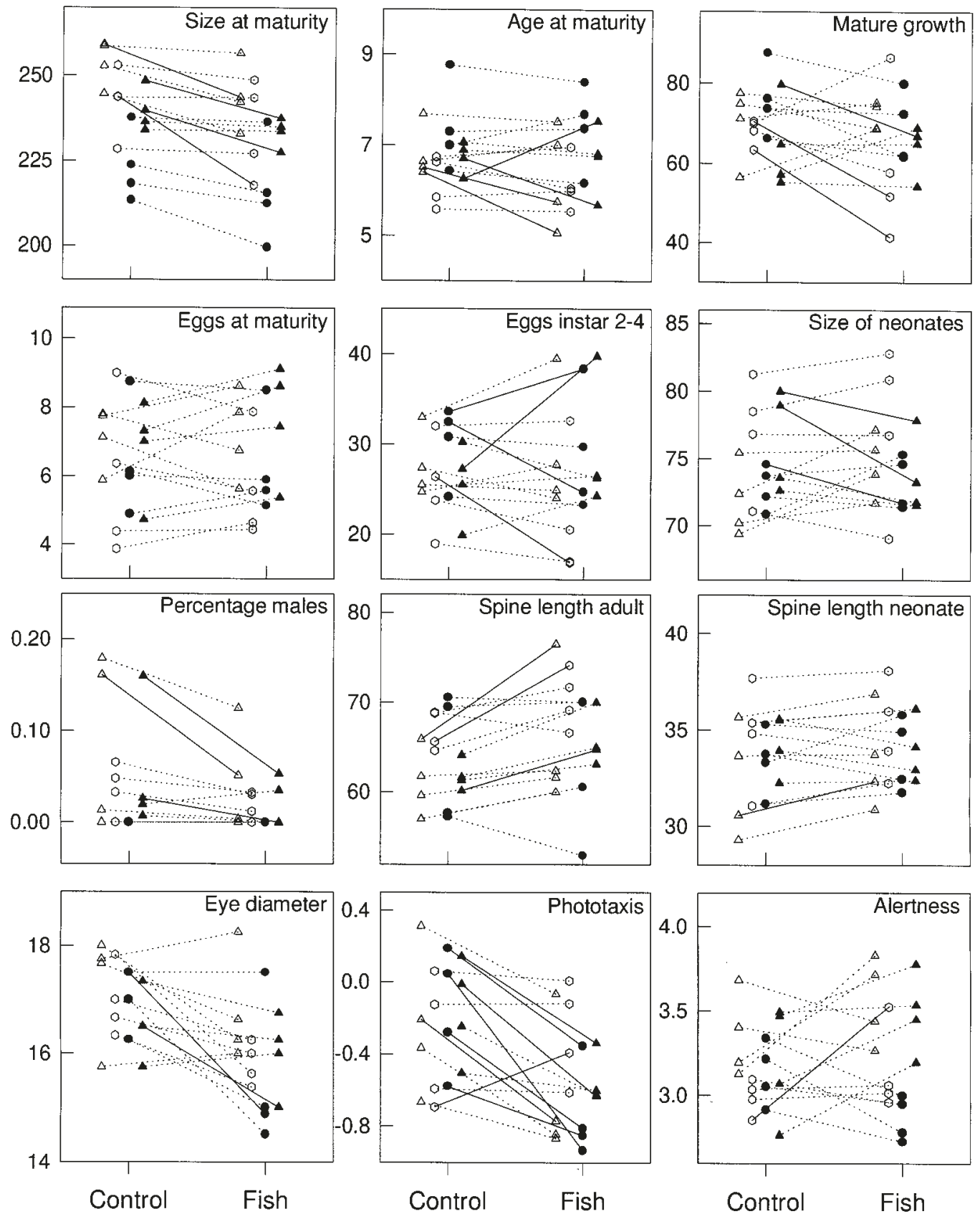

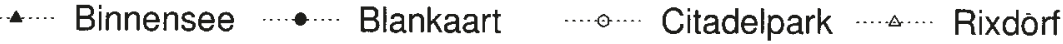

Figure 2: Reaction norms of the 16 clones under study and the 12 traits studied for these clones under conditions with and without the presence of fish chemicals. The units used for the size-related traits are $\mathrm{mm} 100^{-1}$, except for eye diameter, where eyepiece units were used. Age at maturity = days, for phototaxis = the phototactic index (De Meester 1991), and alertness = seconds individual $^{-1}$. Lines with filled symbols indicate clones with a fish background (black triangle $=$ Großer Binnensee; black circle $=$ Lake Blankaart). Lines with open symbols represent clones isolated from fishless locations (white triangle $=$ Rixdorfer Pferdetrdnke; white circle $=$ Citadelpark). Significant $(P<.05)$ differences between fish and nonfish conditions are indicated by solid lines. 


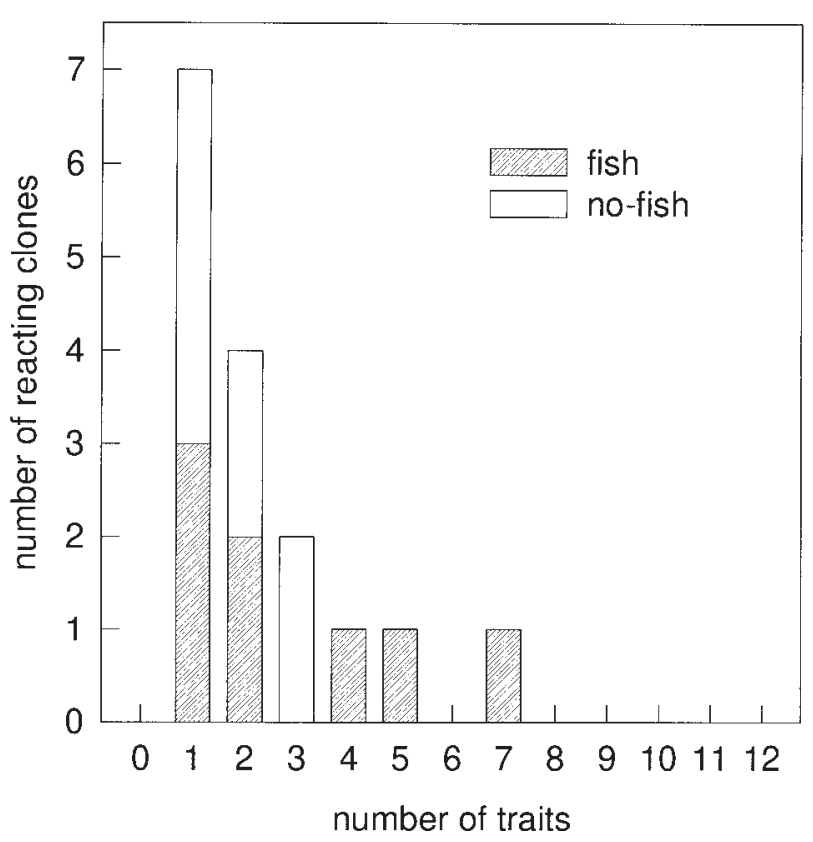

Figure 3: Frequency distribution of the number of clones reacting with a certain number of traits to the presence of fish chemicals. Filled bars indicate clones with a fish background, open bars represent clones isolated from fishless locations. In total, 12 traits were investigated. behavioral and reproduction traits. The third cluster contains both adult and "juvenile" traits. No clear relation between these clusters and the strength of the genetic interactions was observed (cf. fig. 4 and table 2). Interestingly, the overall correlation between the genetic correlations of the traits and the genetic correlations of the changes in these traits equaled .07 , which is obviously not significant. This indicates that the traits and the changes in traits are completely unrelated.

Clones isolated from different habitats could be distinguished statistically in their reaction to the presence of fish chemicals (Wilks's $\lambda=0.21, F=2.77$, df $=30,121$, $P<.001)$. Table 3 shows the squared Mahalanobis distances between the four water bodies and reveals that nonsignificant differences existed only between the animals isolated from Rixdorfer Pferdetränke and from the Großer Binnensee (i.e., the two German populations), and between the Großer Binnensee and Lake Blankaart populations (i.e., the two populations coexisting with fish).

\section{Discussion}

This study shows that clones of the cladoceran species Daphnia magna show a wide variety of responses to the presence of fish chemicals. All clones show at least some reaction, but the number of significant traits varies from one to seven. It is remarkable that no clone was observed that reacted to the presence of fish kairomones with

Table 2: Genetic correlations with significance between selected traits of Daphnia magna

\begin{tabular}{|c|c|c|c|c|c|c|c|c|c|c|c|}
\hline Trait & $\begin{array}{c}\text { Size at } \\
\text { maturity }\end{array}$ & $\begin{array}{l}\text { Age at } \\
\text { maturity }\end{array}$ & $\begin{array}{l}\text { Mature } \\
\text { growth }\end{array}$ & $\begin{array}{l}\text { Eggs at } \\
\text { maturity }\end{array}$ & $\begin{array}{c}\text { Eggs } \\
\text { instar 2-4 }\end{array}$ & $\begin{array}{c}\text { Size of } \\
\text { neonates }\end{array}$ & $\begin{array}{c}\text { Percentage } \\
\text { males }\end{array}$ & $\begin{array}{c}\text { Spine } \\
\text { length } \\
\text { adult }\end{array}$ & $\begin{array}{c}\text { Spine } \\
\text { length } \\
\text { neonates }\end{array}$ & $\begin{array}{c}\text { Eye } \\
\text { diameter }\end{array}$ & Phototaxis \\
\hline Age at maturity & $-.32^{*}$ & & & & & & & & & & \\
\hline Mature growth & -.01 & .35 & & & & & & & & & \\
\hline Number of eggs at maturity & $.13^{*}$ & -.08 & .64 & & & & & & & & \\
\hline Number of eggs instar $2-4$ & $.23^{*}$ & .03 & $.59^{\star *}$ & $.64^{* *}$ & & & & & & & \\
\hline Size of neonates & $.39^{*}$ & $-.20^{*}$ & -.46 & $-.34^{*}$ & -.09 & & & & & & \\
\hline Percentage males & .20 & -.29 & .06 & -.22 & -.13 & .30 & & & & & \\
\hline Spine length adult & $.29^{*}$ & $-.45^{*}$ & .01 & .08 & .12 & $.30^{*}$ & -.14 & & & & \\
\hline Spine length neonate & .29 & .05 & -.17 & .13 & .11 & $.42^{*}$ & -.01 & .20 & & & \\
\hline Eye diameter & -.02 & -.10 & .31 & .14 & .31 & -.29 & .07 & .09 & -.40 & & \\
\hline Phototactic behavior & $.21^{*}$ & $.35^{\star * *}$ & .04 & $.29^{* *}$ & $.16^{* *}$ & -.25 & $-.18^{*}$ & .12 & .01 & -.03 & \\
\hline Alertness & $.29^{*}$ & -.13 & -.07 & $.37^{*}$ & .22 & -.11 & -.03 & .26 & -.18 & .64 & -.14 \\
\hline
\end{tabular}

\footnotetext{
${ }^{\star} P<.05$.

${ }^{* *} P<.01$

${ }^{* * *} P<.001$.
} 


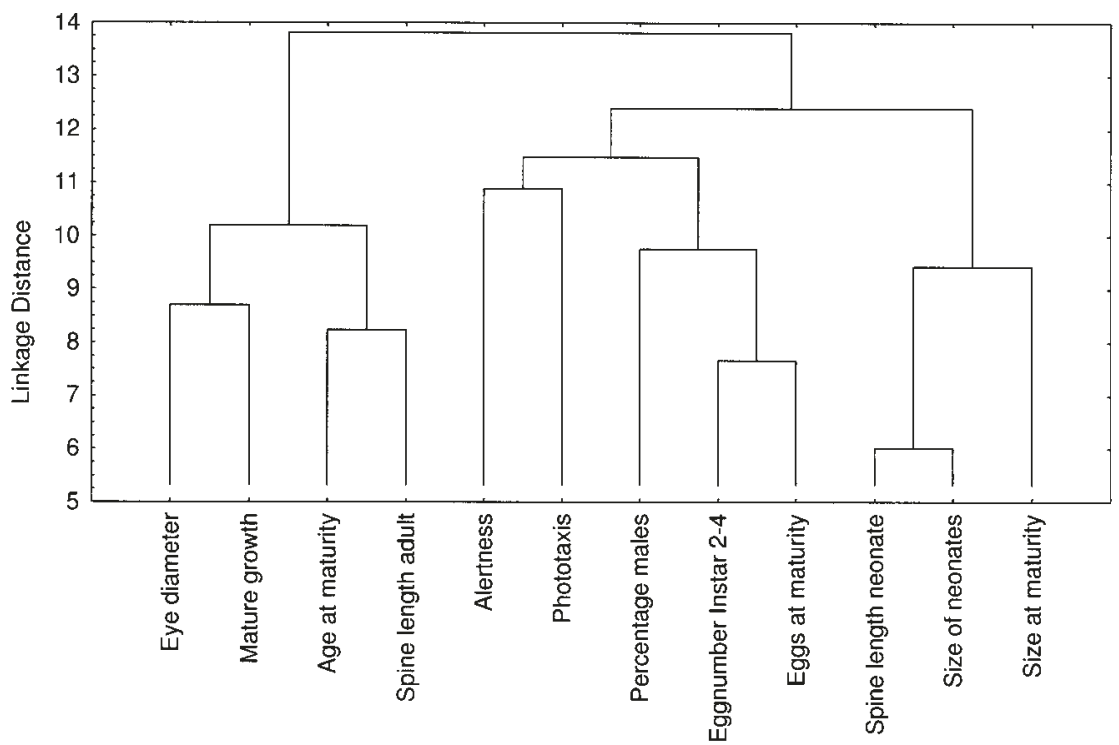

Figure 4: Results of a cluster analysis (complete linkage, Euclidian distances), with the changes in the different traits as a result of the presence/absence of fish kairomones.

changes in all the traits studied. Hence we conclude that the solid line in figure 1 describes the observed reaction patterns of the clones more accurately than the dotted line, although the frequency distribution from figure 3 is certainly skewed to the right.

The lack of strong correlations between the responses to fish kairomones in different traits may reflect a low net benefit of possessing more than one response at a time, either because of a low benefit of showing a combination of induced responses or because of increasing costs of showing multiple responses. The fact that morphological or behavioral responses to fish kairomones are not necessarily associated with changes in life-history traits suggests that the shifts in life history observed in this and other studies are not the result of costs associated with these morphological or behavioral defenses. It is indeed important to differentiate between constraints and adaptive responses. Many authors have expected to see reduced growth or reproductive success in clones showing an induced morphological response as a constraint that reflects the costs of these induced morphological defenses (e.g., Black 1993; Tollrian 1995; Repka and Pihlajamaa 1996). The inability to find correlations between the degree of morphological change and changes in life-history characteristics were interpreted by these authors as evidence for the noncostly nature of morphological defenses in daphnids. Our results, however, indicate that life-history changes are adaptive responses in their own right rather than the result of constraints imposed by the development of other defenses. We argue that there is a lack of necessity to show both responses: if clones react to the presence of predators by forming morphological defenses, the benefit of reacting with other traits may be limited. As was discussed in earlier research (De Meester and Pijanowska 1996), reacting with several traits at the same time can even be nonadaptive at times.

Table 3: Squared Mahalanobis distances between the different habitats

\begin{tabular}{lccc}
\hline & Lake Blankaart & Citadelpark & $\begin{array}{c}\text { Rixdorfer } \\
\text { Pferdetränke }\end{array}$ \\
\hline Großer Binnensee & 4.09 & 6.05 & 3.53 \\
& $(.101)$ & $(.001)$ & $(.069)$ \\
Lake Blankaart & & 6.92 & 8.21 \\
& & $(.007)$ & $(.004)$ \\
Citadel & & 6.15 \\
& & & $(.003)$
\end{tabular}

Note: Numbers in parentheses are significance ( $P$ values). 
Indeed, if clones react to the presence of fish by showing vertical migration behavior, it is not always adaptive to decrease their size at maturity, as food levels in the hypolimnion are typically low and starvation resistance is known to be higher for larger animals (see De Meester 1994).

Only a few studies have computed genetic correlations between traits in daphnids (for a review, see Spitze 1995), and these pertain only to three major types of trade-offs: offspring size versus number, present versus future reproduction, and fecundity versus age at maturity. As indicated by Spitze (1995), computed values for genetic correlations are highly variable, especially for the size versus number of offspring correlations (see also Ebert 1993). To our knowledge, no reports exist about genetic correlations between changes in traits as a result of the presence of predators in daphnids. As a consequence of the way these have to be computed (with random pairing of different individuals and, hence, the combination of measurements in different organisms), we expected and observed that results for such genetic correlations are even more variable still.

Although the clones from the fishless habitats reacted with fewer traits to the presence of fish kairomones, this difference was not significant. It was possible, however, to differentiate between clones isolated from different habitats by discriminant analysis. This differentiation is mainly due to the differences in two traits: phototactic behavior and size of the neonates. In both these traits, clones from habitats in which fish are present tend to respond stronger to the presence of fish chemicals than clones isolated from habitats without fish. This is similar to earlier observations (De Meester 1996) of a pattern of local adaptation for induced changes in phototactic behavior in a set of $30 \mathrm{D}$. magna clones derived from three different natural populations, with clones coexisting with fish showing the stronger reactions to the presence of fish chemicals.

Our results do not indicate that there is a tendency for some clones to react with morphological changes and others with life-history or behavioral changes to the presence of fish chemicals. Different clones showed totally different sets of reactions (see also table 1). The main result of our analysis is, hence, that the reactions of different traits to the presence of predator kairomones are to a large extent uncoupled. Only a few studies have provided data that allow an assessment of these uncoupling phenomena in daphnids. It has been observed (De Meester and Pijanowska 1996) that two behavioral traits were to some extent uncoupled in D. magna, and Spitze (1992) and Lüning (1994) have provided data indicating that neck teeth induction and life-history characteristics are uncoupled in Daphnia pulex. These literature data to- gether with our results suggest that the receptor-effector pathways are very variable, to such an extent that, in the present experiment, nearly every clone showed a different set of reactions to the presence of fish kairomones. This (as was also argued in De Meester and Pijanowska 1996), has important consequences for future studies of the effects of predator-induced changes in prey species. For example, it is clear from our results that most clones can recognize the presence of predator kairomones, even though only eight out of 16 clones responded significantly with a change in phototactic behavior and five out of 16 clones showed a significant change in size at maturity. This implies that when assessing the importance of predator-induced phenotypic shifts, it is not sufficient to investigate only the effect of predator chemicals on one or a few characters of the prey species, but a diverse number of character traits should be studied. It indeed follows from our observations that it is not possible to categorize genotypes as being responsive or not responsive to the presence of predator kairomones; one can only state that a given clone does or does not show a significant shift to the presence of a predator with respect to a particular trait. Still, our results also suggest that phototactic behavior and size at maturity are the traits through which most clones show a response to the presence of fish. These are probably very important traits with respect to vulnerability to fish predation and are thus the better candidates to determine patterns of local adaptation (De Meester 1996).

Antipredator defenses, and the inducibility of such features, have been extensively studied (e.g., Tollrian and Harvell 1998). As stated before, for daphnids, some reports suggest a certain degree of uncoupling of different responses to the presence of predators (Spitze 1992; Black 1993; Lüning 1994; Tollrian 1995; Repka and Pihlajamaa 1996). For other organisms, however, not much is known, although a few authors have suggested that the reaction of different traits might be at least partly uncoupled in such a wide array of species as mayflies (Benton and Pritchard 1990), ciliates (Kusch 1993a), and tobacco (Rickauer et al. 1997).

In other areas of evolutionary ecology, however, the uncoupling of different adaptations has been explored. For example, Rees $(1993,1994)$ showed theoretically that the evolution of iteroparity reduces selection for prolonged seed dormancy. He and others (Hairston and Cáceres 1996) argue that in fact iteroparity and prolonged diapause can be seen as alternative mechanisms to solve the same problem of temporal environmental variations and that there is no reason to have both iteroparity and prolonged diapause. Likewise, Venable and Lawlor (1980) showed that dispersal and prolonged dormancy are alternative ways to adapt to a temporally varying en- 


\section{The American Naturalist}

vironment. Most of the studies cited above have been carried out either using large data sets from the literature, comprising organisms of many different families (Venable and Lawlor 1980; Rees 1993; Hairston and Cáceres 1996) or at least have used multiple species (Kusch 1993a). Our study suggests that, even within species, such alternative mechanisms of dealing with environmental difficulties may exist.

Most theoretical studies and reviews on the subject of predator-induced changes in prey individuals discuss the evolution and the relative fitness of inducible and noninducible genotypes under varying circumstances (Lively 1986; Adler and Harvell 1990; Harvell 1990; van Tienderen 1991). Our results, however, indicate that there is an added complexity to this subject-that is, many genotypes that show different combinations of inducible defenses may coexist in a population. Probably, this coexistence is mediated by the fact that these different combinations of traits result in similar fitness values under circumstances with predators present, as was shown by an earlier study (De Meester et al. 1995), where two clones of Daphnia hyalina $\times$ galeata with different vertical migration behavior and different size at maturity had similar population growth rates under fish predation. The fact that different responses lead to similar fitnesses in a given environment creates space for the maintenance of genetic variation, even for traits that are directly related to the fitness of a given organism.

\section{Acknowledgments}

M.B. was supported by a Max Planck Society (Germany) fellowship and by a fellowship from the European Science Foundation. P.S. was supported by a Max Planck Society (Germany) fellowship. L.D.M. was a postdoctoral researcher with the Fund for Scientific Research (Belgium) and was supported by a travel grant from the Max Planck Society, by grants NR 2.0128.94 and G 0260.97 from the Fund for Scientific Research, and grant OT/96/ 13 from the Katholieke Universiteit Leuven. We thank H. Boriss, N. G. Hairston, Jr., C. D. Harvell, W. Lampert, K. Spitze, and L. J. Weider for their comments on earlier versions of the manuscript.

\section{Literature Cited}

Adler, F. R., and C. D. Harvell. 1990. Inducible defenses, phenotypic variability and biotic environments. Trends in Ecology \& Evolution 5:407-410.

Benton, M. J., and G. Pritchard. 1990. Mayfly locomotory responses to endoparasitic infection and predator presence: the effects on predator encounter rate. Freshwater Biology 23:363-372.

Black, A. R. 1993. Predator-induced phenotypic plasticity in Daphnia pulex: life history and morphological responses to Notonecta and Chaoborus. Limnology and Oceanography 38:986-996.

Boersma, M. 1995. The allocation of resources to reproduction in Daphnia galeata: against the odds? Ecology 76:1251-1261.

De Meester, L. 1991. An analysis of the phototactic behaviour of Daphnia magna clones and their sexual descendants. Hydrobiologia 225:217-227.

. 1993. Genotype, fish-mediated chemicals, and phototactic behavior in Daphnia magna. Ecology 74: 1467-1474.

. 1994. Life histories and habitat selection in Daphnia: divergent life histories of D. magna clones differing in phototactic behaviour. Oecologia (Berlin) 97:333-341.

- 1996. Evolutionary potential and local genetic differentiation in a phenotypically plastic trait of a cyclical parthenogen, Daphnia magna. Evolution 50: 1293-1298.

De Meester, L., and J. Pijanowska. 1996. On the traitspecificity of the response of Daphnia genotypes to the chemical presence of a predator. Pages 407-417 in P. H. Lenz, D. K. Hartline, J. E. Purcell, and D. L. Macmillan, eds. Zooplankton: sensory ecology and physiology. Gordon \& Breach, Amsterdam.

De Meester, L., L. J. Weider, and R. Tollrian. 1995. Alternative antipredator defences and genetic polymorphism in a pelagic predator-prey system. Nature (London) 378:483-485.

Ebert, D. 1993. The trade-off between offspring size and number in Daphnia magna: the influence of genetic, environmental, and maternal effects. Archiv für Hydrobiologie Supplement 90:453-473.

Gliwicz, Z. M., and A. Jachner. 1993. Lake restoration by manipulating the behaviour of planktivorous fish with counterfeit information on risk to predation? Internationale Vereinigung für Theoretische und Angewandte Limnologie Verhandlungen 25:666-670.

Hairston, N. G., Jr., and C. E. Cáceres. 1996. Distribution of crustacean diapause: micro- and macroevolutionary pattern and process. Hydrobiologia 320:27-44.

Harvell, C. D. 1990. The ecology and evolution of inducible defenses. Quarterly Review of Biology 65:323-340.

Hessen, D. O., and E. van Donk. 1993. Morphological changes in Scenedesmus induced by substances released from Daphnia. Archiv für Hydrobiologie 127:129-140.

Hobæk, A., and P. Larsson. 1990. Sex determination in Daphnia magna. Ecology 71:2255-2268.

Jacobs, J. 1967. Untersuchungen zur Funktion und Evolution der Zyklomorphose bei Daphnia mit besonderer Berücksichtigung der Selektion durch Fische. Archiv für Hydrobiologie 62:467-541.

Kusch, J. 1993a. Behavioural and morphological changes 
in ciliates induced by the predator Amoeba proteus. Oecologia (Berlin) 96:354-359.

- 1993b. Induction of defensive morphological changes in ciliates. Oecologia (Berlin) 94:571-575.

Kvam, O. V., and O. T. Kleiven. 1995. Diel horizontal migration and swarm formation in Daphnia in response to Chaoborus. Hydrobiologia 307:177-184.

Lampert, W. 1991. The dynamics of Daphnia magna in a shallow lake. Internationale Vereinigung für Theoretische und Angewandte Limnologie Verhandlungen 24:795-798.

Lampert, W., K. O. Rothhaupt, and E. von Elert. 1994. Chemical induction of colony formation in a green alga (Scenedesmus acutus) by grazers (Daphnia). Limnology and Oceanography 39:1543-1550.

Larsson, P., and S. Dodson. 1993. Invited review-chemical communication in planktonic animals. Archiv für Hydrobiologie 129:129-155.

Lively, C. M. 1986. Canalization versus developmental conversion in a spatially variable environment. American Naturalist 128:561-572.

Lüning, J. 1994. Anti-predator defenses in Daphnia-are life-history changes always linked to induced neck spines? Oikos 69:427-436.

- 1995. Life-history responses to Chaoborus of spined and unspined Daphnia pulex. Journal of Plankton Research 17:71-84.

Macháček, J. 1995. Inducibility of life history changes by fish kairomone in various developmental stages of Daphnia. Journal of Plankton Research 17:1513-1520.

Meyer, J. S., C. G. Ingersoll, L. L. McDonald, and M. S. Boyce. 1986. Estimating uncertainty in population growth rates: jackknife versus bootstrap techniques. Ecology 67:1156-1166.

Nilsson, P. A., C. Brönmark, and L. B. Pettersson. 1995. Benefits of a predator-induced morphology in crucian carp. Oecologia (Berlin) 104:291-296.

Parejko, K., and S. I. Dodson. 1991. The evolutionary ecology of an antipredator reaction norm: Daphnia pulex and Chaoborus americanus. Evolution 45:16651674.

Pijanowska, J. 1994. Fish-enhanced patchiness in Daphnia distribution. Internationale Vereinigung für Theoretische und Angewandte Limnologie Verhandlungen 25:2366-2368.

Reede, T. 1995. Life history shifts in response to different levels of fish kairomones in Daphnia. Journal of Plankton Research 17:1661-1667.

Rees, M. 1993. Trade-offs among dispersal strategies in British plants. Nature (London) 366:150-152.

- 1994. Delayed germination of seeds: a look at the effects of adult longevity, the timing of reproduction, and population age-stage structure. American Naturalist 144:43-64.
Repka, S., and K. Pihlajamaa. 1996. Predator-induced phenotypic plasticity in Daphnia pulex-uncoupling morphological defenses and life history shifts. Hydrobiologia 339:67-71.

Repka, S., M. Walls, and M. Ketola. 1995. Neck spine protects Daphnia pulex from predation by Chaoborus, but individuals with longer tail spine are at a greater risk. Journal of Plankton Research 17:393-403.

Rice, W. R. 1989. Analyzing tables of statistical tests. Evolution 43:223-225.

Rickauer, M., W. Brodschelm, A. Bottin, C. Veronesi, H. Grimal, and M. T. Esquerre Tugaye. 1997. The jasmonate pathway is involved differentially in the regulation of different defence responses in tobacco cells. Planta (Heidelberg) 202:155-162.

Ringelberg, J. 1991. Enhancement of the phototactic reaction in Daphnia hyalina by a chemical mediated by juvenile perch (Perca fluviatilis). Journal of Plankton Research 13:17-25.

Ślusarczyk, M. 1995. Predator-induced diapause in Daphnia. Ecology 76:1008-1013.

Spitze, K. 1992. Predator-mediated plasticity of prey life history and morphology-Chaoborus americanus predation on Daphnia pulex. American Naturalist 139: 229-247.

-1995. Quantitative genetics of zooplankton life histories. Experientia (Basel) 51:454-464.

Spitze, K., J. Burnson, and M. Lynch. 1991. The covariance structure of life-history characters in Daphnia pulex. Evolution 45:1081-1090.

Stearns, S. C. 1989. The evolutionary significance of phenotypic plasticity. BioScience 39:436-445.

Stemberger, R. S., and J. J. Gilbert. 1987. Defenses of planktonic rotifers against predators. Pages 227-239 in W. C. Kerfoot and A. Sih, eds. Predation: direct and indirect impacts on aquatic communities. University Press of New England, Hanover, N.H.

Stibor, H. 1992. Predator induced life-history shifts in a freshwater cladoceran. Oecologia (Berlin) 92:162165.

Swaffar, S. M., and W. J. O’Brien. 1996. Spines of Daphnia lumholtzi create feeding difficulties for juvenile bluegill sunfish (Lepomis macrochirus). Journal of Plankton Research 18:1055-1061.

Threlkeld, S. T. 1976. Starvation and the size structure of zooplankton communities. Freshwater Biology 6:489496.

Tollrian, R. 1990. Predator-induced helmet formation in Daphnia cucullata (Sars). Archiv für Hydrobiologie 119:191-196.

- 1995. Predator-induced morphological defenses: costs, life history shifts, and maternal effects in Daphnia pulex. Ecology 76:1691-1705.

Tollrian, R., and C. D. Harvell, eds. 1998. The evolu- 


\section{The American Naturalist}

tion of inducible defenses. Princeton University Press, Weider, L. J., and J. Pijanowska. 1993. Plasticity of DaphPrinceton, N.J.

van Tienderen, P. H. 1991. Evolution of generalists and specialists in spatially heterogeneous environments. Evolution 45:1317-1331.

Venable, D. L., and L. Lawlor. 1980. Delayed germination and dispersal in desert annuals: escape in space and time. Oecologia (Berlin) 46:272-282. nia life histories in response to chemical cues from predators. Oikos 67:385-392.

Zaret, T. M., and W. C. Kerfoot. 1975. Fish predation on Bosmina longirostris: body-size selection versus visibility selection. Ecology 56:232-237.

Associate Editor: C. Drew Harvell 